\title{
Decreasing cost effectiveness of testing for latent TB in HIV in a low TB incidence area
}

\author{
Santino Capocci ${ }^{1}$, Colette Smith ${ }^{2}$, Stephen Morris ${ }^{3}$, Sanjay Bhagani ${ }^{4}$, \\ Ian Cropley ${ }^{4}$, Ibrahim Abubakar $^{5,6}$, Margaret Johnson $^{1}$ and Marc Lipman ${ }^{1,7}$ \\ Affiliations: \\ ${ }^{1}$ Department of HIV and Thoracic Medicine, Royal Free London NHS Foundation Trust, London, UK. \\ ${ }^{2}$ Department of Infection and Population Health, University College London, London, UK. \\ ${ }^{3}$ Department of Applied Health Research, University College London, London, UK. \\ ${ }^{4}$ Department of Infectious Diseases, Royal Free London NHS Foundation Trust, London, UK. \\ ${ }^{5}$ Research Department of Infection and Population Health, University College London, London, UK. \\ ${ }^{6}$ Medical Research Council Clinical Trials Unit, London, UK. \\ ${ }^{7}$ Division of Medicine, University College London, London, UK. \\ Correspondence: \\ Santino Capocci, Department of Thoracic Medicine, Royal Free London NHS Foundation Trust, Pond Street, \\ London, NW3 2QG, UK. \\ E-mail: santino.capocci@nhs.net
}

ABSTRACT Testing for latent tuberculosis infection (LTBI) in HIV-infected persons in low tuberculosis (TB) incidence areas is often recommended. Using contemporary, clinical data, we report the yield and cost-effectiveness of testing all HIV attendees, two current UK strategies and no LTBI testing.

Economic modelling was performed utilising 10-year follow up data from a large HIV clinical cohort. Outcomes were numbers of cases of active TB and incremental cost per quality-adjusted life year (QALY) gained.

Between 2000 and 2010, 256 people were treated for TB/HIV co-infection. 72 (28\%) occurred $\geqslant 3$ months after HIV diagnosis and may have been prevented by LTBI testing. Between 2000 and 2005, the incremental cost per QALY gained for the British HIV Association (BHIVA) and UK National Institute of Care Excellence (NICE) strategies, and testing all clinic attendees was $€ 6270, € 6998$ and $€ 33$ 473, respectively. These rose to $€ 9332$, €32564 and $€ 74067$, respectively, between 2005 and 2010. Probabilistic sensitivity analysis suggested that at a threshold of $€ 24000$ per additional QALY, the most cost-effective strategies would be NICE or testing all in 2000-2005 and BHIVA during 2005-2010.

Both UK testing regimens missed cases but are cost-effective compared with no testing. Using recent data, they all became more expensive, suggesting that alternative or more targeted TB testing strategies must be considered.

@ERSpublications

Targeted latent TB testing strategies in HIV are cost effective, but lately have become more expensive http://ow.ly/JnWjU

This article has supplementary material available from erj.ersjournals.com.

Received: April 082014 | Accepted after revision: Jan 072015 | First published online: April 162015

Conflict of interest: Disclosures can be found alongside the online version of this article at erj.ersjournals.com 


\section{Introduction}

The increased risk of developing active tuberculosis (TB) attributable to HIV in low TB incidence countries is estimated to be around 40-times that of background rates [1,2]. Preventive treatment of latent TB infection (LTBI) with 6-9 months of isoniazid reduces the risk of re-activation by around two-thirds, though may result in adverse events and drug-drug interactions $[2,3]$. The incidence of active TB in the UK is not declining at the same rate as in other western European nations, and HIV co-infection likely contributes to this [4]. Given the high risk of reactivation, treatment of individuals with latent TB infection is also important for TB elimination in Europe [5-9]. Hence, testing and treatment for LTBI in people living with HIV has been recommended by many European national guideline bodies, the British HIV Association (BHIVA), the UK National Institute for Health and Care Excellence (NICE) and the European AIDS Clinical Society (EACS) [5, 10-12]. The UK guidelines were both published in 2011, but their testing protocols differ.

Risk factors for developing active TB disease in the UK include blood CD4 count $<500$ cells $\mu \mathrm{L}^{-1}$ and being of black African ethnicity [13]. Many European countries test all HIV clinic attendees, either with a tuberculin skin test (TST) or interferon- $\gamma$ release assays (IGRA). NICE recommends more targeted testing for LTBI in all individuals with HIV infection and blood CD4 count $<500$ cells $\mu \mathrm{L}^{-1}$. Because of the discordance between the TST and IGRA in those with low CD4 cell counts, they suggest using both IGRA and TST if the CD4 is $<200$ cells $\mu \mathrm{L}^{-1}$; and IGRA with or without concurrent TST between 200 and 500 cells $\mu \mathrm{L}^{-1}$. BHIVA guidance advises testing with IGRA alone depending on country of origin, blood CD4 count and duration of anti-retroviral therapy (ART), then treating all those with a positive IGRA (fig. 1). These strategies have not previously been compared for cost-effectiveness.

Sustained ART appears to reduce progression to active TB by up to two-thirds [14]. Therefore, as the use of ART becomes more widespread, the risk of reactivation in HIV-infected people with LTBI is likely to fall. In addition, HIV population demographics in the UK are changing [15], with less migration from areas of higher HIV and TB prevalence. This may also impact on rates of TB.

Health economic assessments frequently guide national planning and policy. Their predictive accuracy depends on their ability to model factors that contribute to future need and may change over time. Here, we compare the utility and cost-effectiveness of testing all HIV clinic attendees with a single IGRA, and the NICE and BHIVA latent TB testing strategies using HIV clinic data obtained during two consecutive time periods: 2000-2005 and 2005-2010. These were selected as the use of ART was increasing and national HIV demographics changing in our low TB incidence region. The analysis used incremental cost per case averted (ICCA - the extra cost required by one strategy compared to another to avert one case of active tuberculosis) and incremental cost per quality-adjusted life year (QALY) gained.

\section{Methods}

Setting

The Royal Free Hospital (London, UK) provides care for a large HIV infected, stable population. Around one-third of patients treated here originate from sub-Saharan Africa. The background prevalence of HIV in London is 525 per 100000 adults aged 16-59 years [16]; the incidence of active TB local to the hospital is 36.8 per 100000 [17].

All patients attending our HIV service from 2000 to 2010 were included, other than those with a TB diagnosis before, or within 3 months of, their HIV diagnosis. Three months was selected to ensure that all subjects under follow up would have adequate time to be assessed for LTBI prior to any subsequent diagnosis of active TB.

\section{Data source}

The Royal Free Hospital HIV Database contains information prospectively gathered from all patients receiving care. Between 2000 and 2010, testing and treatment for latent TB was not performed routinely in people with HIV infection. We modelled BHIVA and NICE strategies using clinical and demographic data from 2000-2005 and 2005-2010 to assess the costs and benefits compared with no testing, with each other and testing all patients with a single IGRA. Costs were measured in terms of testing for LTBI, preventive treatment and treating active TB cases. Benefits were measured in terms of prevented active TB cases and QALY. We calculated the cost-effectiveness of each strategy using incremental cost per QALY gained and ICCA. In the 2000-2005 model, all patients eligible for testing under BHIVA or NICE guidance were tested based on the first CD4 count in 2000. Those not eligible at this time would be followed at each subsequent CD4 count (as a surrogate for an HIV clinic appointment, usually every 3-6 months) and would be tested as soon as they satisfied the relevant guideline criteria (fig. 1). CD4 follow-up continued until the end of December 2004 and cases of TB recorded. The same analysis was performed using 20052010 data. 

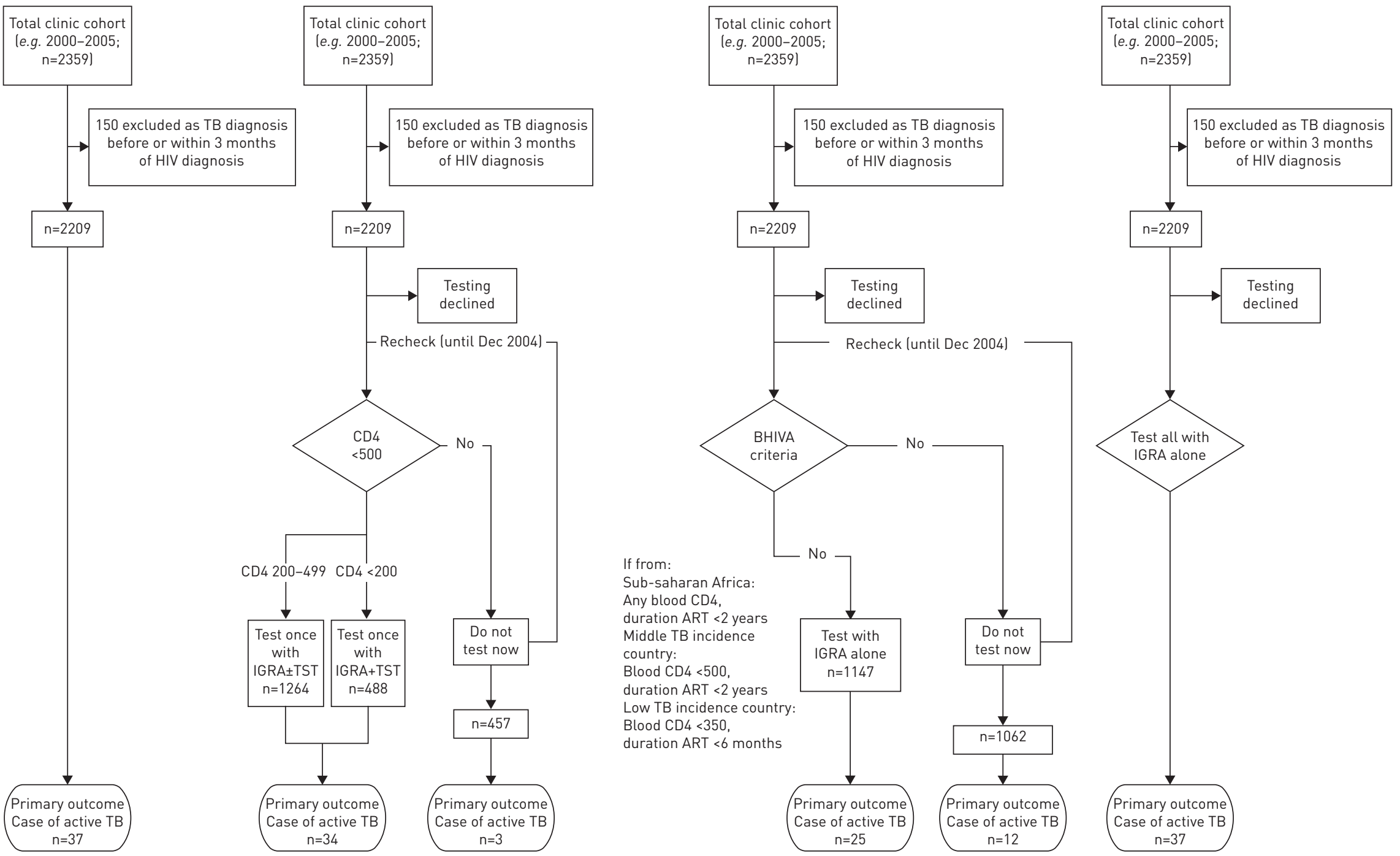

FIGURE 1 Testing algorithms for no testing, National Institute for Health and Care Excellence (NICE) strategy, British HIV Association (BHIVA) strategy, and testing all clinic attendees. Data from 2000-2005 [10, 11]. Blood CD4 cell counts are presented as cells. $\mu \mathrm{L}^{-1}$. TB: tuberculosis; IGRA: interferon- $\gamma$ release assay; TST: tuberculin skin test; ART: anti-retroviral therapy. 


\section{Model structure and assumptions}

We assumed the following: all HIV-positive patients would be eligible except those excluded because of a diagnosis of active TB before or within 3 months of HIV diagnosis; initial testing would occur in $87 \%$ of those eligible [18]; subjects were tested on one occasion, but $3 \%$ would have indeterminate IGRA results, leading to one repeat test [19]; TST/IGRA positivity rates were estimated to be $13 \%$ in subjects originating from a country in sub-Saharan Africa, 10\% from middle TB incidence countries and 3\% from low TB incidence countries [18]; sensitivity of an IGRA was 91\% [20]; uptake of latent TB preventive treatment was $87 \%$ with $62 \%$ effectiveness using six months of isoniazid $[3,18]$; and QALY reductions for active and latent $\mathrm{TB}$ were -0.676 and -0.007 respectively, using previously published estimates for this age group with active TB disease (see section $\mathrm{A}$ in the online supplementary material) [11].

Costs were measured using an English National Health Service perspective in 2011/2012 UK£ (with an exchange rate of $€ 1=£ 0.83$ ). We measured the costs of IGRA, TST, six months treatment with isoniazid and treatment for active TB. The cost of ART in subjects with TB/HIV coinfection who had no other indication for starting ART was not included. Unit costs were based on published sources and local hospital charges (table s1 in section A of the online supplementary material). The time horizon was lifelong, although TB follow-up data were available for 5 years. Costs and benefits were discounted at 3.5\% per year [21].

\section{Cost-effectiveness}

Cost-effectiveness was measured in terms of the ICCA and the incremental cost per QALY gained over each 5-year period; and calculated separately for the time periods (2000-2005, 2005-2010). We conducted incremental analyses of the BHIVA strategy versus no testing, NICE versus BHIVA strategies and testing all attendees versus NICE. The cost-effectiveness ratios were calculated as the difference in costs between the two comparators divided by the difference in outcomes (active TB cases, QALYs).

\section{Sensitivity analysis}

We undertook univariate and multivariate deterministic sensitivity analyses; and varied costs for IGRA, TST, plus treatment of latent and active TB to reflect the additional expense of increased TB-related morbidity, isoniazid-induced hepatotoxicity or TB drug resistance. We varied the uptake of testing, sensitivity of IGRA, uptake and effectiveness of isoniazid preventive therapy using published data. The effectiveness varied between $40 \%$ (an estimate of protection given non-optimal adherence) and $100 \%$ (a figure approached in studies in high-resource, low TB incidence areas) $[18,22]$. The impact on quality of life was varied for LTBI preventive treatment and active TB treatment. Multivariate most-costly and least-costly scenarios were also considered (section A in the online supplementary material).

We also used a probabilistic sensitivity analysis (PSA) with 10000 Monte Carlo simulations of the model. From this, we computed uncertainty ranges for point estimates of cost-effectiveness and cost-effectiveness acceptability curves, showing the probability that each option was cost-effective for different cost-effectiveness thresholds. The PSA accounted simultaneously for uncertainty in uptake, IGRA sensitivity, incidence of TB, detection rate of each strategy, costs and quality of life years lost for TB infection. The distributions and parameter values used are given in section A in the online supplementary material (table s1).

Ethical approval was previously granted for prospective data extraction from clinical notes.

\section{Results}

The Royal Free HIV clinic cohort consisted of 1136 individuals in 2000, 2025 in 2005 and 2461 in 2010. 689 (61\%) were on ART consisting of three drugs in 2000, 1505 (74\%) in 2005 and 2119 (86\%) in 2010.

\section{Cases of active tuberculosis with HIV infection}

Between 2000 and 2010, there were 256 cases of tuberculosis with HIV co-infection at this centre. 72 (28\%) had a TB diagnosis $\geqslant 3$ months after their HIV diagnosis. Of these, 38 (53\%) had culture confirmed $\mathrm{TB}$, sensitive to all first line anti-tuberculosis drugs, one of isoniazid resistant $\mathrm{TB}$ and one of multidrug resistant $\mathrm{TB}$.

\section{Cases not prevented by testing guidelines}

Both BHIVA and NICE strategies would not have tested all people that would later develop active tuberculosis. Compared with BHIVA, NICE's broader criteria predicted more cases consistently (fig. 1), although costs were higher. Five of the six cases not tested by NICE were already taking ART or had interrupted ART. Two of the six were UK born. 22 of the 31 cases not eligible using BHIVA criteria were UK born, and 28 were using ART or had interrupted ART when diagnosed with active TB. 
Comparative cost effectiveness: 2000-2005 and 2005-2010

Between 2000 and 2005, 37 patients known to be HIV positive and undergoing care at the Royal Free Hospital developed active tuberculosis. Using BHIVA criteria, 1147 subjects would be tested over 5 years and 12 of 37 (32.4\%; 95\% CI 18.0-49.8\%), who later developed tuberculosis would not have been tested. Using the NICE testing model, 1752 subjects would have been tested, including 34 of the 37 patients with TB disease (8.1\% not tested; 95\% CI 1.7-21.9\%). Allowing for $87 \%$ uptake of testing, the cost of testing all eligible subjects over 5 years, plus treating those with latent tuberculosis as well as the active TB cases missed by testing, would be $€ 378713$, €395825 and €419928 using BHIVA and NICE guidance and testing all clinic attendees respectively (table 1). These equate to an extra €171 (BHIVA), €179 (NICE), $€ 190$ (all) in costs for each person attending the HIV clinic. Compared with no testing, 6.4 QALYs would be gained with BHIVA guidance, 8.9 with NICE and 9.6 testing all. Comparing BHIVA to no testing, NICE to BHIVA, and all to NICE, the incremental cost-effectiveness ratios were $€ 6270$ (95\% uncertainty range $€ 3482-€ 7864)$, €6998 (€4130-€18813) and €33 473 (€6895-€164519) respectively (table 2).

Using 2005-2010 data, 35 patients developed tuberculosis. Using BHIVA criteria, 1240 subjects would be tested and 11/35 (31.4\%; 95\% CI 17.4-49.4) who later developed TB would not have been tested. Using NICE guidance, 2228 subjects would have been tested including 30/35 subjects with subsequent TB (14.3\% not tested, 95\% CI 5.4-31.1\%). Discounted QALYs gained by testing were 6.4 with BHIVA, 8.2 with NICE and 8.8 testing all and the incremental costs per QALY gained (95\% uncertainty range) were €9332 (€5396-€11958), €32564 (€25066-€66251) and €74067 (€19625-€665734) (table 2).

\section{Sensitivity analysis}

LTBI testing became more cost effective as the cost of an IGRA fell, the uptake or effectiveness of LTBI-preventive treatment increased, or the cost of treatment for active tuberculosis rose. The IGRA indeterminate rate made little difference. If an IGRA cost €30, LTBI-preventive treatment was 98\% effective, or treatment of one case of active TB increased to $€ 14000$, testing for LTBI between 2000 and 2005 using any strategy became cost saving compared with no testing.

The cost effectiveness acceptability curves, derived from the probabilistic sensitivity analysis, are shown in figures 2 and 3 for the time periods 2000-2005 and 2005-2010, respectively. These indicate that if a health service were willing to pay an extra $€ 24000$ ( $£ 20000$ ) for an additional QALY [21], the NICE strategy would most probably be best value between 2000 and 2005, and the BHIVA strategy between 2005 and 2010 (figures 2 and 3).

\section{Discussion}

\section{Principal findings}

At an incremental cost per QALY gained cost-effectiveness threshold of $€ 24000-€ 36000$, testing and treating latent tuberculosis in people with HIV (using ART in the majority of cases) appears to be cost-effective with

TABLE 1 Costs of no testing, testing using BHIVA, testing using NICE strategies and testing all clinic attendees

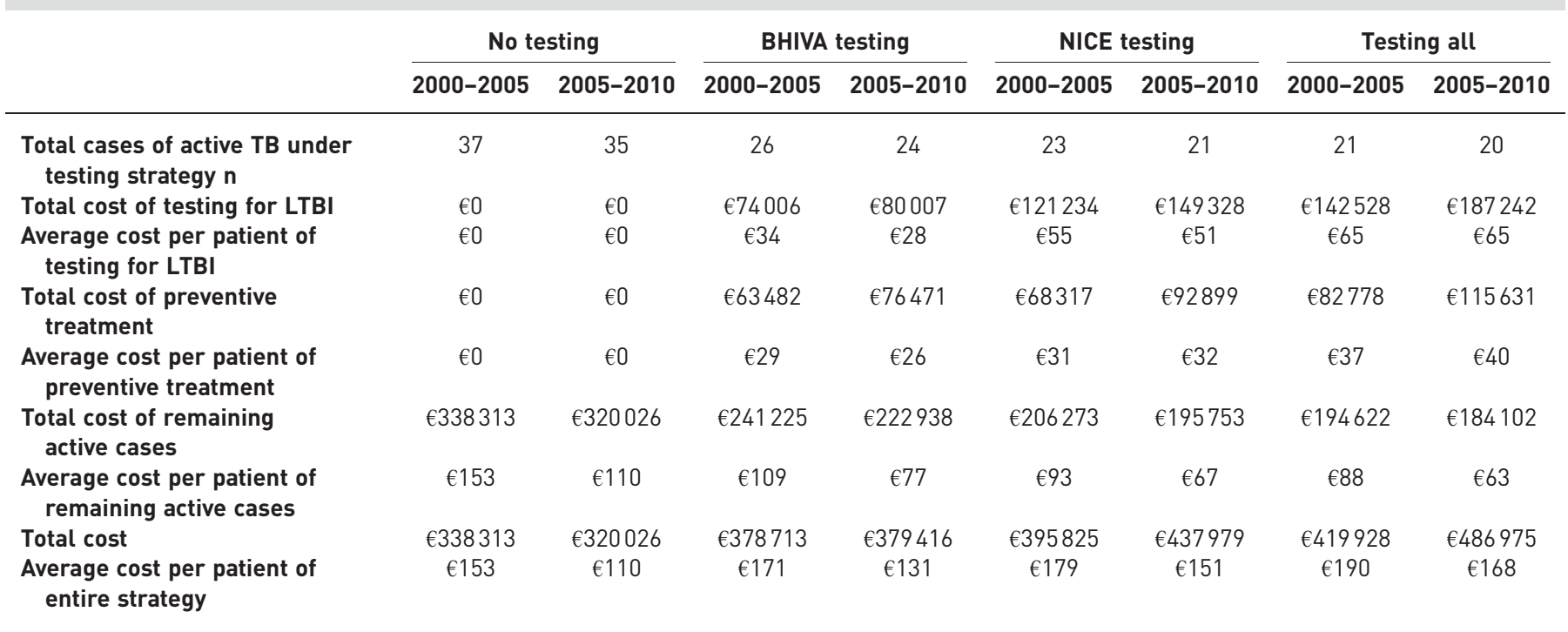

Data from 2000-2005 (n=2209) and 2005-2010 (n=2902). BHIVA: British HIV Association; NICE: National Institute for Health and Care Excellence; LTBI: latent tuberculosis infection. 
TABLE 2 Comparative testing comparing BHIVA strategy to no testing, NICE strategy to BHIVA strategy and testing all attendees to NICE

\begin{tabular}{|c|c|c|c|c|c|c|}
\hline & \multicolumn{2}{|c|}{ BHIVA versus no testing } & \multicolumn{2}{|c|}{ NICE versus BHIVA } & \multicolumn{2}{|c|}{ All versus NICE } \\
\hline & 2000-2005 & 2005-2010 & $2000-2005$ & 2005-2010 & 2000-2005 & 2005-2010 \\
\hline \multicolumn{7}{|l|}{ Difference in costs } \\
\hline Cost of testing & $€ 74006$ & $€ 80007$ & $€ 47228$ & $€ 41835$ & $€ 21294$ & $€ 37914$ \\
\hline Cost of preventive treatment & $€ 63482$ & $€ 76471$ & $€ 4836$ & $€ 16428$ & $€ 14460$ & $€ 22732$ \\
\hline Cost of treating active TB & $-€ 97088$ & $-€ 97088$ & $-€ 34952$ & $-€ 27185$ & $-€ 11651$ & $-€ 11651$ \\
\hline $\begin{array}{l}\text { Total cost (95\% uncertainty } \\
\text { range) }\end{array}$ & $€ 40400(€ 10245-€ 106741)$ & $€ 59389(€ 16000-€ 156057)$ & $€ 17112(€ 5323-€ 63871)$ & $€ 58564(€ 21768-€ 152475)$ & $€ 24104$ (€4667-€65 032) & $€ 48996(€ 14351-€ 118383)$ \\
\hline Extra cost per patient in clinic & $€ 18.29$ & $€ 20.47$ & $€ 7.75$ & $€ 20.18$ & $€ 10.91$ & $€ 16.88$ \\
\hline \multicolumn{7}{|l|}{$\begin{array}{l}\text { Difference in outcomes } \\
\text { discounted at } 3.5 \% \text { per year } \\
\text { (95\% uncertainty ranges) }\end{array}$} \\
\hline Cases of active TB prevented & $10(7.5-15)$ & $10(7.6-14)$ & $4(3.3-4.3)$ & $3(2.4-3.4)$ & $1(0.6-1.8)$ & $1(0.6-1.8)$ \\
\hline QALYs gained & $6.44(1.8-12.5)$ & $6.36(2.0-12.1)$ & $2.45(1.3-3.6)$ & $1.80(1-2.6)$ & $0.72(0.5-0.8)$ & $0.66(0.5-0.9)$ \\
\hline \multicolumn{7}{|l|}{$\begin{array}{l}\text { Cost effectiveness, discounted } \\
\text { at } 3.5 \% \text { per year } \\
\text { (95\% uncertainty ranges) }\end{array}$} \\
\hline $\begin{array}{l}\text { Incremental cost per case } \\
\text { prevented }\end{array}$ & $€ 3997$ (€1329-€7395) & $€ 5875(€ 2049-€ 10981)$ & $€ 4550$ (€1760-€17695) & $€ 19888(€ 9958-€ 52746)$ & $€ 19803$ (€2619-€91 518) & $€ 40241$ (€7797-€176378) \\
\hline $\begin{array}{l}\text { Incremental cost per } \\
\text { QALY gained }\end{array}$ & $€ 6270(€ 3482-€ 7864)$ & $€ 9332$ (€5396-€11 958) & $€ 6998$ (€4130-€18813) & $€ 32564(€ 25066-€ 66251)$ & $€ 33473$ (€6895-€164519) & $€ 74067$ (€19625-€665 734) \\
\hline
\end{tabular}




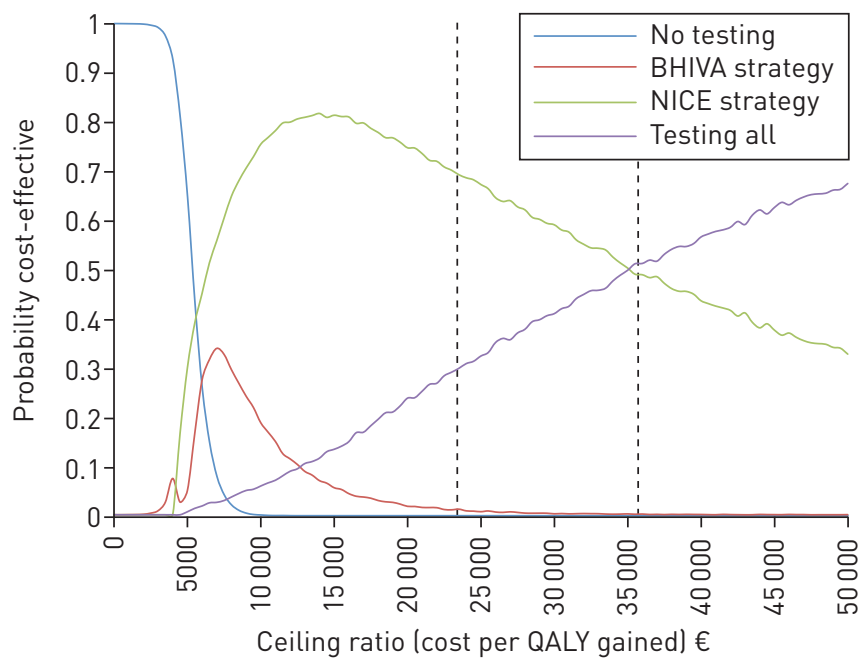

FIGURE 2 Cost effectiveness acceptability curves for No testing, British HIV Association (BHIVA) and National Institute for Health and Care Excellence (NICE) algorithms and testing all clinic attendees, 2000-2005. Using the probabilistic sensitivity analysis from 2000 to 2005 data, there is a $68 \%$ probability that the NICE strategy will be cost-effective where the health service is willing to pay a maximum of $€ 24000$ ( $£ 20000)$ per quality-adjusted life year (QALY) gained (first vertical dashed line).

respect to no testing in a low TB incidence, high-resource setting [21]. Of the strategies compared, testing based on NICE criteria was the most cost-effective option using 2000-2005 data at a cost-effectiveness threshold of $€ 24000$ per QALY and testing all clinic attendees at $€ 36000$ per QALY (fig. 2). Between 2005 and 2010, the BHIVA strategy was the most likely cost-effective option at a threshold of $€ 24000$ and NICE at $€ 36000$. Testing all clinic attendees became considerably less cost effective in this time period (fig. 3). This change may have resulted from altering UK HIV demographics (e.g. as there were proportionally fewer HIV-infected individuals in the UK from TB-endemic countries) or from an increase in ART usage, which would reduce the risk of progression from LTBI to active TB $[15,23]$. For example, one-third of our clinic population now have a CD4 cell count $<500$ cells $\mu \mathrm{L}^{-1}$ (unpublished data). If these changes continue and are representative of other low TB incidence settings, testing may become much less cost effective in future irrespective of the strategy selected.

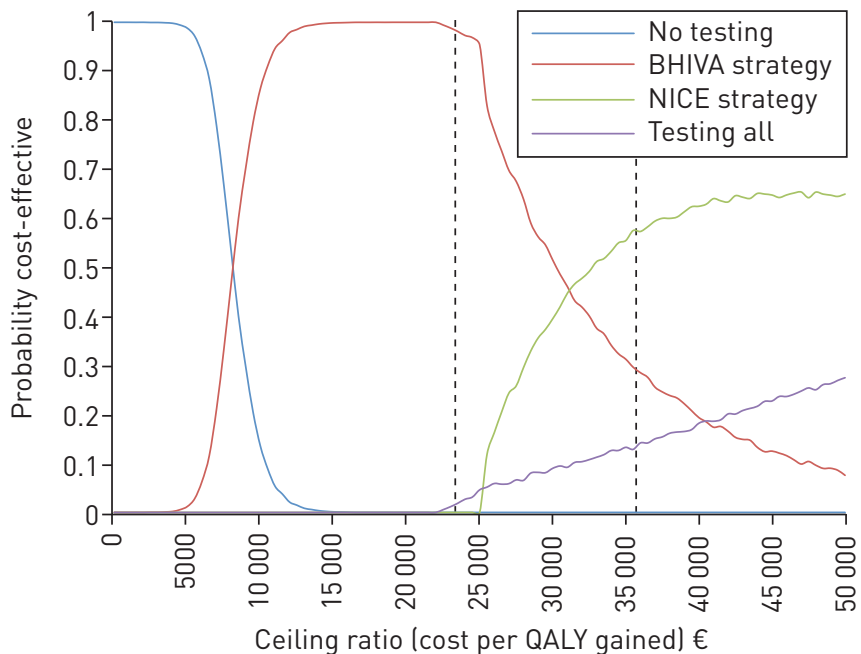

FIGURE 3 Cost effectiveness acceptability curves for No testing, British HIV Association (BHIVA) and National Institute for Health and Care Excellence (NICE) algorithms and testing all clinic attendees, 2005-2010. Using the probabilistic sensitivity analysis from 2005 to 2010 data, there is a $97 \%$ probability that the BHIVA strategy will be cost-effective where the health service is willing to pay a maximum of $€ 24000$ ( $£ 20000)$ per QALY gained (first vertical dashed line) and $58 \%$ probability that the NICE strategy will be cost-effective at maximum $€ 36000$ ( $£ 30000)$ per quality-adjusted life year (QALY) gained (second vertical dashed line). 
It is important to note that even with optimal implementation, our data also indicate that these testing strategies would prevent only around one-quarter of recorded cases of active tuberculosis; as the majority of patients with HIV co-infection are of unknown HIV status when they present with TB and are then subsequently diagnosed with HIV. This is consistent with national reports [23]. These patients tend to access HIV care late, have a lower blood CD4 count and higher disease associated morbidity and hence treatment costs [23]. Hence, a more effective strategy may involve community-based HIV testing, and then, if positive, subsequent assessment for TB.

\section{Limitations, criticisms and assumptions}

Our study has several limitations. We sought to assess the cost-effectiveness of testing and treating for LTBI, yet it is not a prospective, controlled trial comparing isoniazid preventive therapy and placebo in people living with HIV and a positive TST or IGRA. Most cost-effectiveness evaluations seek to predict what will happen in the future, and thus guide healthcare policy. Here we report the impact of testing now and over the last 10 years. By using real world data from two 5-year analyses, we can compare external influences on the utility and cost-benefits of testing. We believe that this is important, as our findings suggest that the drivers of cost-effectiveness are changing rapidly; hence, modelling analyses that predict future trends without acknowledging and measuring this variation may be inaccurate. Our study does not imply that the effects of ART and isoniazid preventive treatment only last for 5 years; and these will likely be of considerable benefit for longer in this setting.

A number of assumptions are contained within our model. Costings use the available literature for TB cases without HIV co-infection, even though some studies suggest that there is an increased morbidity and mortality and a higher rate of adverse events and drug-drug interactions associated with HIV [24, 25]. This may drive up treatment costs and decrease quality of life in those with active tuberculosis, which further justifies systematic LTBI testing. HIV care in resource-rich areas involves regular follow up and laboratory testing. Preventive treatment for opportunistic infection (such as Pneumocystis or toxoplasmosis) is an established concept in this setting, and we might expect LTBI testing and treatment to be easier to implement in people already receiving HIV care than in new arrivals or TB contacts. A further advantage of treating LTBI would be to prevent onward TB transmission, although the cost-utility of this has not been included in our model.

The costs of hepatotoxicity related to preventive TB treatment and active TB treatment have not been specifically determined, though were included within the sensitivity analysis. In our model, an IGRA cost of $€ 72$ ( $£ 60$ ), the hospital list price for a T-Spot.TB test (Oxford Immunotec, Oxford, UK), was used. Actual costs for this assay may be less and could also fall using the QuantiFERON Gold In-Tube (Qiagen, Venlo, The Netherlands). It is not clear if the two tests have equivalent sensitivity or indeterminate result rates in this population [26-28].

QALY reductions from TB cases without HIV coinfection were used. This is supported by recent work showing a similar loss in quality of life measured by EQ-5D in those with active drug-sensitive TB with or without HIV coinfection [29]. Our analysis has been from a clinical perspective and has only used the potential costs and health status in those subjects tested. We have not included potential cases prevented in contacts and their costs, either pecuniary or in quality of life. From a public health position, the appropriateness of a $€ 24000-36000$ per QALY or $€ 15000-23000$ per case averted ceiling could be questioned.

\section{Implementation}

Given the very high risk of progression to active disease, treating LTBI in HIV seems an obvious strategy to reduce TB incidence. Previous studies have reported difficulties in implementation, both at a clinic and patient level. In the Swiss Cohort Study, a programme to treat latent TB resulted in only 37\% of HIV patients with a positive TST receiving a full course of such therapy. In part, this was due to physicians' poor adherence to the recommendation [22]. In those that were given preventive treatment, however, there were no subsequent cases of active $\mathrm{TB}$, a finding that has been mirrored in more recent studies from Western Europe [18, 30]. Our assumptions of uptake and LTBI treatment completion are based on contemporary studies from London [18]; however, by using results from a meta-analysis on TST-positive HIV-infected individuals, comparing LTBI treatment against placebo, we may have underestimated the efficacy of LTBI treatment in people with a positive IGRA (as modelled here) and hence the cost-effectiveness of testing $[3,18]$. As technologies to detect LTBI improve, the potential for a test with a better positive predictive value for the development of active TB would further reduce the costs and morbidity associated with LTBI treatment. 


\section{Conclusion}

Data from this study show that testing for tuberculosis infection in those with a known HIV diagnosis seems cost-effective using either NICE or BHIVA strategies, both of which were published in 2011 and draws on data from 1996 to 2006. Using our clinic data from 2000 to 2005, the NICE strategy is simple, less likely to miss cases and cheaper to implement than universal testing. This has reduced the cost-effectiveness of testing. Checking for latent TB in known HIV-infected persons cannot impact on the majority of cases of TB-HIV co-infection who are diagnosed with both conditions simultaneously. This argues for more population-based HIV testing, with subsequent TB risk assessment and treatment if found to be infected [23].

Our work indicates that a prospective, health economic study of TB testing in HIV infected subjects living in resource rich, low TB incidence countries is warranted.

\section{References}

1 Antonucci G, Girardi E, Raviglione MC, et al. Risk factors for tuberculosis in HIV-infected persons. JAMA 1995; 274: 143 .

2 Badri M, Wilson D, Wood R. Effect of highly active antiretroviral therapy on incidence of tuberculosis in South Africa: A cohort study. Lancet 2002; 359: 2059-2064.

3 Akolo C, Adetifa I, Shepperd S, et al. Treatment of latent tuberculosis infection in HIV infected persons. Cochrane Database Syst Rev 2010: CD000171.

4 Tuberculosis in the UK: 2014 report. London: Public Health England. www.gov.uk/government/publications/ tuberculosis-tb-in-the-uk Date last updated: Sept 25, 2014. Date last accessed: Nov 1, 2014.

5 D'Ambrosio L, Dara M, Tadolini M, et al. Tuberculosis elimination: Theory and practice in Europe. Eur Respir J 2014; 43: 1410-1420.

6 Voniatis C, Migliori GB, Voniatis M, et al. Tuberculosis elimination: dream or reality? The case of Cyprus. Eur Respir J 2014; 44: 543-546.

7 Diel R, Loddenkemper R, Zellweger JP, et al. Old ideas to innovate tuberculosis control: preventive treatment to achieve elimination. Eur Respir J 2013; 42: 785-801.

8 van der Werf MJ, Sandgren A, D'Ambrosio L, et al. The European Union standards for tuberculosis care: do they need an update? The European Union standards for tuberculosis care: Do they need an update? Eur Respir J 2014; 43: 933-942.

9 European Centre for Disease Prevention and Control. Progressing towards TB elimination. Stockholm, ECDC, 2010.

10 Pozniak AL, Coyne KM, Miller RF, et al. British HIV Association guidelines for the treatment of TB/HIV coinfection 2011. HIV Med 2011; 12: 517-524.

11 NICE. Tuberculosis - Clinical diagnosis and management of tuberculosis, and measures for its prevention and control. NICE Clinical Guideline 117, 2011. Available from: www.nice.org.uk/guidance/cg117/resources/guidancetuberculosis-pdf Date last accessed: Jan 17, 2012.

12 European AIDS Clinical Society Guidelines for the diagnosis and treatment of resistant and latent TB in HIV positive persons 2014. Available from: www.eacsociety.org/files/guidelines_english_71_141204.pdf Date last accessed: July 15, 2014.

13 Grant AD, Bansi L, Ainsworth J, et al. Tuberculosis among people with HIV infection in the United Kingdom: Opportunities for prevention? AIDS 2009; 23: 2507-2515.

14 Golub JE, Saraceni V, Cavalcante SC, et al. The impact of antiretroviral therapy and isoniazid preventive therapy on tuberculosis incidence in HIV-infected patients in Rio de Janeiro, Brazil. AIDS 2007; 21: 1441-1448.

15 Health Protection Agency. HIV in the United Kingdom: 2011 Report. London: Health Protection Services, Colindale. November 2011. Available from: http://webarchive.nationalarchives.gov.uk/20140629102648/http://www. hpa.org.uk/webc/HPAwebFile/HPAweb_C/1317131685847 Date last accessed: June 12, 2012. Date last updated: Jan20, 2012.

16 HIV prevalence greatest in London boroughs but quality of care is high. Health Protection Agency, Colindale November 2010. Available from: http://webarchive.nationalarchives.gov.uk/20140714084352/http://www.hpa.org. uk/ProductsServices/LocalServices/London/LondonPressReleases/lond101126HIVprevalencegreatestinLondon/ Date last updated: June 15, 2011. Date last accessed: June 18, 2012.

17 Health Protection Agency. HPA HPP single map for rate of tuberculosis diagnoses per 100,000 population, Camden; London. Available from: http://profiles.hpa.org.uk/IAS/dataviews/report?reportId=4\&viewId=7\&geoId=8 \&geoSubsetId=\&select=00AG Date last accessed: July 10, 2012.

18 Kall MM, Coyne KM, Garrett NJ, et al. Latent and subclinical tuberculosis in HIV infected patients: a cross-sectional study. BMC Infect Dis 2012; 12: 107

19 Brock I, Ruhwald M, Lundgren B, et al. Latent tuberculosis in HIV positive, diagnosed by the M. tuberculosis specific interferon-gamma test. Respir Res 2006; 7: 56

20 Aichelburg MC, Rieger A, Breitenecker F, et al. Detection and prediction of active tuberculosis disease by a whole-blood interferon-gamma release assay in HIV-1-infected individuals. Clin Infect Dis 2009; 48: 954-962.

21 National Institute for Health and Clinical Excellence. Guide to the methods of technology appraisal. Guide to the methods of technology appraisal. 2008, Jun; Available from: www.nice.org.uk/media/b52/a7/tamethodsguideupdated june2008.pdf Date last updated: Jun 2008. Date last accessed: Feb 21, 2013.

22 Elzi L, Schlegel M, Weber R, et al. Reducing tuberculosis incidence by tuberculin skin testing, preventive treatment, and antiretroviral therapy in an area of low tuberculosis transmission. Clin Infect Dis 2007; 44: 94-102.

23 Rice B, Elford J, Yin Z, et al. Decreasing incidence of tuberculosis among heterosexuals living with diagnosed HIV in England and Wales. AIDS 2013; 27: 1151-1157.

24 Lanternier F, Dalban C, Perez L, et al. Tolerability of anti-tuberculosis treatment and HIV serostatus. Int J Tuberc Lung Dis 2007; 11: 1203-1209.

25 Dean GL, Edwards SG, Ives NJ, et al. Treatment of tuberculosis in HIV-infected persons in the era of highly active antiretroviral therapy. AIDS 2002; 16: 75-83. 
26 Mandalakas AM, Hesseling AC, Chegou NN, et al. High level of discordant IGRA results in HIV-infected adults and children. Int J Tuberc Lung Dis 2008; 12: 417-423.

27 Talati NJ, Seybold U, Humphrey B, et al. Poor concordance between interferon-gamma release assays and tuberculin skin tests in diagnosis of latent tuberculosis infection among HIV-infected individuals. BMC Infect Dis 2009; 9: 15 .

28 Stephan C, Wolf T, Goetsch U, et al. Comparing QuantiFERON-tuberculosis gold, T-SPOT tuberculosis and tuberculin skin test in HIV-infected individuals from a low prevalence tuberculosis country. AIDS 2008; 22: 2471-2479.

29 Kittikraisak W, Kingkaew P, Teerawattananon Y, et al. Health related quality of life among patients with tuberculosis and HIV in Thailand. PLoS One 2012; 7: e29775.

30 Clark SA, Martin SL, Pozniak A, et al. Tuberculosis antigen-specific immune responses can be detected using enzyme-linked immunospot technology in human immunodeficiency virus (HIV)-1 patients with advanced disease. Clin Exp Immunol 2007; 150: 238-244. 